\section{"BLISTER PACKS" RATHER THAN "ASHES TO ASHES, DUST TO DUST" FOR MEDICATIONS}

To the editor:

It is laudable that we as community have finally lapped up to the idea of disposing of "old, unused, unwanted, or expired" (OUUE) medications. ${ }^{1} \mathrm{How}-$ ever, we must ponder that how come we have reached this point where OUUE medications have assumed the nuisance value of epidemic proportions. Is it because there are always alternative pharmaceuticals to explore for fine tuning our over-demanding healthcare needs dictated by direct-to-consumer advertisements of pharmaceuticals swarming mass media? Is it because we have become oblivious to the costs that our over-demanding needs incur during the fine tuning of our healthcare wherein our focus on our insurance premiums and healthcare co-pays fails to notice exorbitant costs of our healthcare reimbursed by our third party payers? Is it because feasible doctor shopping allows our successful search for physicians who are more agreeable than others to reach common ground regarding our over-demanding healthcare needs especially when our pharmaceutical prescriptions are being discussed?

Instead of proclaiming the new-age adage: "It's complicated" to overlook our problem, we should invoke an age-old adage: "Prevention is better than cure" as our solution. Futuristically, it can be worthwhile to explore replacing pill bottles overfilled with medications by prescriptions of just sufficient blisterpackaged medications filling and refilling personalized automated medication dispensing systems located inside patients' homes. ${ }^{2-4}$ The exploration of this futuristic preventative solution may nullify the questions raised by our current "complicated" solutions. Can incineration or alternatively landfilling of OUUE medications collected during drug take-back programs be environmentally perfect methods ever?5,6 Won't lapping up the model of "prescribing less and dispensing lesser" force us to shell out more co-pays for our medication fills and refills during our more frequent visits to our pharmacies on the lines of our regular visits to our food supermarkets with or without the options for home deliveries therein as well?

While primarily-indoor-limited living in spaces with artificially controlled environmental moistures and temperatures might have allowed proliferation of manageable pill bottles in temperate climate, nonindoor-limited living in spaces with poorly controlled environmental moistures and temperatures may have always warranted medication dispensing in blisterpackaging to protect and sustain the potency of dispensed medications in tropical climate. ${ }^{7}$ Although blister-packaged medications may appear easily returnable to pharmacies and thereafter easily reusable for dispensing by pharmacies, the potency and safety of medications cannot be ensured in the hands of the patient populations. Therefore, the environment safety initiative to futuristically curtail OUUE medications warrants blister-packaged medications to be filled and refilled in personalized automated medication dispensing systems located inside patients' homes so that OUUE medications within personalized automated medication dispensing systems located inside patients' homes can be directly retrieved by dedicated home-pharmacy personnel who can ensure medications' chain-of-custody before considering such medications' potential for safe dispensing among future patients.

Summarily, even though it seems too futuristic to have blister-packaged medications replacing all pillbottle medications and avoiding incineration or landfilling of OUUE medications as "ashes to ashes, dust to dust," we are hoping to make a case for an ecofriendly future by replacing epidemic proportions of OUUE medications dispensed in pill bottles with just sufficient blister-packaged medications filling and refilling personalized automated medication dispensing systems located inside patients' homes because in the long run, environmental costs of OUUE medications are bound to soar as compared to the costs of initiatives curtailing them.

Deepak Gupta, MD Clinical Assistant Professor Department of Anesthesiology Wayne State University Detroit, Michigan

Shushovan Chakrabortty, MD, PhD Clinical Assistant Professor Department of Anesthesiology Wayne State University Detroit, Michigan 


\section{REFERENCES}

1. US Food \& Drug Administration: Disposal of Unused Medicines: What You Should Know. Available at bttps:// www.fda.gov/drugs/safe-disposal-medicines/disposal-unusedmedicines-what-you-should-know. Accessed September 21, 2019 .

2. ROPACK: Benefits of Blister Packaging for Emerging Markets. Available at https://www.ropack.com/2014/04/10/benefits-ofblister-packaging-for-emerging-markets/Accessed September 21, 2019

3. US Food \& Drug Administration: Statement on FDA's request for information on requiring fixed-quantity blister packaging for certain opioid pain medicines to help decrease unnecessary exposure to opioids. Available at https://www.fda.gov/newsevents/press-announcements/statement-fdas-request-informationrequiring-fixed-quantity-blister-packaging-certain-opioid-pain Accessed September 21, 2019.

4. MedGadget: HealthOneMed Introduces Dispense-A-Pill Personal Medication Manager. Available at bttps://www. medgadget.com/2011/03/healthonemed_introduces_dispense apill_personal_medication_manager.html Accessed September 21, 2019
5. Texas Commission on Environmental Quality. Study of the Methods for Disposing of Unused Pharmaceuticals. Available at https://www.tceq.texas.gov/assets/public/comm_exec/pubs/sfr/09 8.pdf Accessed September 21, 2019.

6. ACS Publications: Life Cycle Comparison of Environmental Emissions from Three Disposal Options for Unused Pharmaceuticals. Available at https://pubs.acs.org/doi/suppl 10.1021/es203987b/suppl_file/es203987b_si_001.pdf Accessed September 21, 2019.

7. Packaging Insights: Don't sweat it: Amcor instills blister packs with extra moisture protection for hot climate. Available at https://www.packaginginsights.com/news/dont-sweat-itamcor-instills-blister-packs-with-extra-moisture-protectionfor-hot-climate.html Accessed September 21, 2019. 